\title{
Segmentation of Multi-Tumor from PET/CT Images
}

\author{
K.Shruthi, V.Vivekitha, S. Syed Althaf, G. Gnancy Subha
}

\begin{abstract}
The aim of the project is to develop a methodology for automatic segmentation of multiple tumor from PET/CT images. Image pre-processing methods such as Contrast Limited Adaptive Histogram Equalization (CLAHE), image sharpening and Wiener filtering were performed to remove the artifacts due to contrast variations and noise. The image was segmented using K-means, Threshold segmentation, watershed segmentation, FCM clustering Segmentation, Mean shift Clustering Segmentation, Graph Cut Segmentation. Evaluation was made for the segmentation against the Ground Truth. Various Features was selected and extracted. Classification was made using SVM classifier and KNN classifier to classify the tumor as benign or malignant. The proposed method was carried out using PET/CT images of lung cancer patients and implemented using MATLAB.
\end{abstract}

\section{Index Terms-CLAHE, Graph cut, MATLAB.}

\section{INTRODUCTION}

In U.S and worldwide lung cancer is the main cause for cancer death and it is the second most common disease after heart disease. Small cell lung cancers(SCLC) and non-small cell lung cancer are the two lung cancers. The stage of cancer depends on how it spread in the body. Chemotheraphy, Targeted theraphy, immunotheraphy, surgery and radiation theraphy are the mostly used therapeutic methods for the treatment of cancer.

Doctors cannot predict the cancers until its advanced stage. Early identification of cancer is very difficult because the symptoms can be identified in the advanced stage.

PET/CT is the most preferred modality, interpretation of large dataset by radiologist seems to be time consuming and laborious which leads to misclassify. The aim of this paper is to develop a methodology for automatic detection of cancer from PET/CT images.

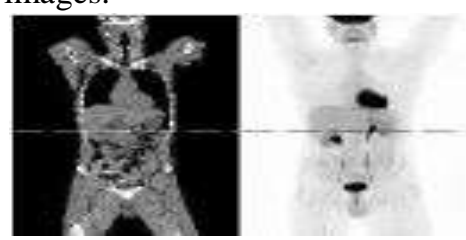

Figure 1.1 CT and PET

$\mathrm{PET} / \mathrm{CT}$ is the combination of PET and CT. CT will give

Revised Manuscript Received on 14 September, 2019.

K.Shruthi, Assistant professor, Department of Biomedical Engineering, St.Peter's Institute of Higher Education and Research, Chennai, Tamilnadu, India.

V.Vivekitha, Assistant professor, Department of Biomedical Engineering, Velalar College of Engineering and Technology, Erode.

S. Syed Althaf, Assistant professor, Department of Biomedical Engineering, St.Peter's Institute of Higher Education and Research, Chennai, Tamilnadu, India.

G. Gnancy Subha, Assistant professor, Department of Biomedical Engineering, Salem College of Engineering and Technology, Salem, Tamilnadu, India. the detailed anatomical map of interior body. Positron Emission Tomography will shows the function of an organ. Combining PET and CT will gives the detailed map of body's metabolism. Figure 1.1 shows the CT and PET image. In CT image, soft tissue is seen as black and the bone is white. PET slice gives the radioactive radiation in which the scale goes from low to high radiation.

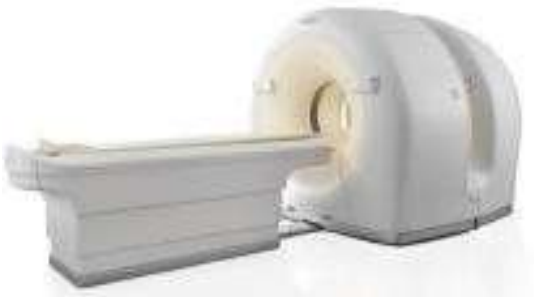

Figure 1.2 PET/CT

The patient is slided inside through a cylinder. In PET/CT machine the PET and CT are placed next to each other which is shown in figure 1.2. Scan takes 45 minutes. The fluroine18 is a radioactive which decays to the stable isotope oxygen18 by the cmission of positron. This positron annihilates together with an electron and forms two gamma photons which moves in opposite direction. These gamma photons will detect concentration of FDG in a patient.

\section{LITERATURE REVIEW}

Appadurai Ramalingam et al., (2016) proposed a method for detection of multi tumor from CT-DICOM images. In this CT Image In DICOM Format is filtered using Median Filter 5x5x5 Mask. Unsharp Masking was used to Sharpen the Image after that thresholding performed to eliminate unwanted tissues with High and Low Intensities. Morphological Closing and Opening operation was carried to eliminate the artifacts due to thresholding. Normal Tissues are eliminated by Dilation, Complementation, Intersection. Segmentation was performed using 3D Labelling Algorithm. 350 CT DICOM images are used, Sensitivity of this method is $93.3 \%$ and the Specificity is $90 \%$.

Srikanth B. et al., (2016) proposed a a system for detection of multi tumor from MRI image. MRI image in DICOM format was filtered using Median filter to remove noise. Feature containing irregular tissues are pulled out. For the removed clusters thresholding was applied. Features such as local contrast, entropy, valuable correlation was extracted using GLCM. Classification was performed by combination of SVM and fuzzy c-means. Finally Performance was analysed by calculating basis of time, tumor area, PSNR, RI, VOI. 
Gawade Prathamesh et al., (2016) proposed a method for detection of lung cancer cells. First the image was changed from RGB to Gray scale image. The image was sharpened using High pass filter and the Median Filter was used to remove noise. Segmentation was made by Thresholding followed by Otsu Watershed algorithm. This watershed algorithm was applied only to gray scale. Binary Morphological operation was used to access the sectioned structure.

Ning Guo et al., (2015) proposed a system for lung cancer diagnosis using SVM. In this feature selection process other than SUV mean, CT texture features such as CT value, gray level, entropy, uniformity, Tumor heterogeneity was calculated. Global moran I(d) was applied to characterize intra-tumor heterogeneity which measures spatial auto correlation and then the features were stored. SVM classifier was used for classification. Performance was evaluated using leave one out cross validation and ROC curve was used to characterise the performance for different feature selection such as Heterogeneity and SUV mean; Hetrogeneity, SUV mean and CT Texture; Heterogeneity. Of these features, they had concluded that Heterogeneity was the best feature for classification.

Wei Ju, Deihui Xiang et al., (2015) proposed a system for segmentation of lung tumor. Anatomical and Functional images was extracted using Anisotropic diffusion Filter. Random Walk was conducted on PET image. Down hill region was detected in PET images and used as Initial seed for CT images. Graph cut segmentation was performed by Maximum flow algorithm. Performance of segmentation was analyzed using Dice Similarity Coefficient against Ground truth. It is stated that Graph cut method has small cut problem. Hence, combination of Random walk and graph cut was used.

Zexi Yu et al., (2015) proposed a method for automated segmentation of tumor. It was stated that Random walk is Good against noise and weak boundaries but limited to deal with Multi focal uptake regions. Hence, Graph cut method was used for energy minimization problem by Maximum flow algorithm. The seeds are placed only on background not for foreground. tsource and the tsink value was set as 0.4 and 0.1 respectively. Performance was evaluated using ground truth and evaluation matrix such as similarity indices and hausdroff distance was performed. Comparison was made with thresholding.

Punithavathy K. et al., (2015) developed an algorithm for automatic detection of lung cancer. In Preprocessing, CLAHE was used to enhance the local contrast of image and wiener filter of $3 \times 3$ to remove the noise. Performance metrics such as MSE and PSNR was calculated to analyse the performance of preprocesssing filters. ROI was extracted using Morphological closing operators. Feature is extracted using GLCM. Segmentation was performed using otsu's global thresholding and the classification was made using FCMalgorithm. Out of 888 samples, 761 samples was correctly classified and the accuracy of this system was $92.67 \%$.

Neha Bangara et al., (2014) proposed a method for detedction of tumor by color segmentation. The color image was not converted as grayscale. The method worked directly on RGB image. 2D Median filter was used to eliminate random noise. ROI was selected using GUI Polygon method. Feature extraction was performed on selected region by Calculating area, equi-Disatance, Perimeter of the tumor. Segmentation was performed using Binary tree quantization clustering algorithm and finally it was compared with K-means clustering algorithm.

Nivetha P. et al., (2014) proposed a methodology for detection of lung cancer at an early stage. Histogram equalization was used to enhance the contrast of image. Median filtering was performed to remove Glare, Noise present in an image. Segmentation was made by using Optimal thresholding. Feature selection was selected using genetic algorithm. The selected features were extracted using GLCM and finally the Classification was made by SVM linear classifier

Sunil Kumar et al., (2014) developed 3-class FCM algorithm with thresholding which is noise efficient. The proposed 3-Class FCM method improves efficiency of image by modifying the initialization of fuzzy petition matrix this method uses normally distributed pseudorandom numbers generator with Gaussian distribution for initial estimates of petition matrix. The thresholding with FCM is used to generate the logical segmented images. Finally, the colour segments are generated by utilizing the results of FCM. And the results of the proposed FCM method are tested upon the variety of medical images and compared with the widely used Global thresholding and Otsu's method.

Yang Gui et al., (2012) proposed an algorithm by incorporating the advantages of mean shift (MS) segmentation and spectral clustering (SC) method, the proposed approach provides effective and robust segmentation. Firstly, input image is transformed from a pixel-based to a region-based model by using the MS algorithm. The input image after MS segmentation is composed of multiple disjoint regions that preserve the desirable discontinuity characteristics of the image. Then the regions are treated as nodes in the image plane and a graph structure is applied to represent them. The final step is to apply the improved SC to perform globally optimal clustering. To avoid some incorrect partitioning when considering each region as one graph node, we assign different numbers of nodes to represent the regions according to area ratios among the regions.

Mohamed Ben Salah et al., (2011) proposed a methodology for multiregion graph cut image segmentation in a kernel-induced space. The method consists of minimizing a functional containing an original data term which references the image data transformed via a kernel function. After that the kernel function is optimized by iterating in two consecutive steps, graph cut optimization and fixed point iterations for updating the regions parameters. A quantitative and comparative performance was made over a very large number of experiments on synthetic images illustrated the flexibility and effectiveness of the proposed method. The proposed approach yields competitive results in comparison with using the correct

Published By:

Blue Eyes Intelligence Engineering

\& Sciences Publication 
model learned in advance. The flexibility and effectiveness of the method were tested over various types of real images like synthetic SAR images, medical and natural images.

\section{METHODOLOGY \& RESULTS}

\subsection{BLOCK DIAGRAM}

Figure 3.1 shows the steps involved in the project. The image is first preprocessed using varies filters and then segmented using varies types of segmentation methods. Feature values are extracted from the segmented image and it is trained using classifier. The testing is made against the training dataset and the classification made as benign or malignant.

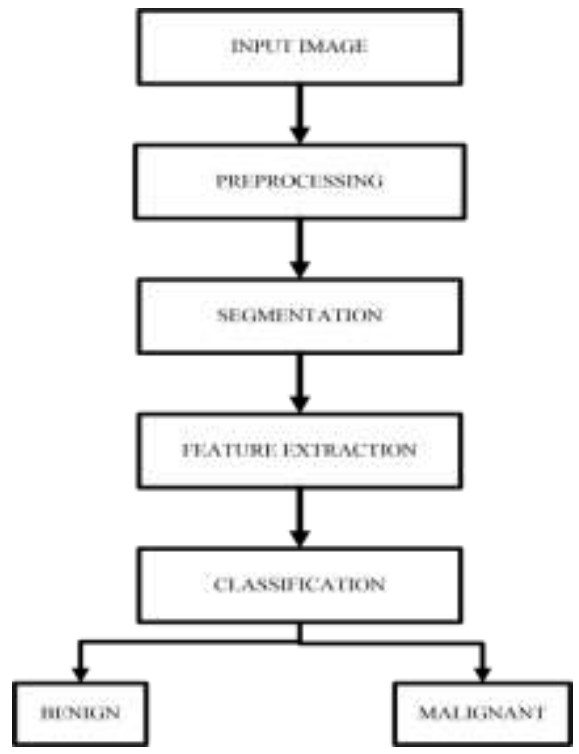

Figure.3.1 Workflow of the project

\section{RESULTS AND DISCUSSION}

\subsection{PREPROCESSING}

\subsubsection{Filter.}

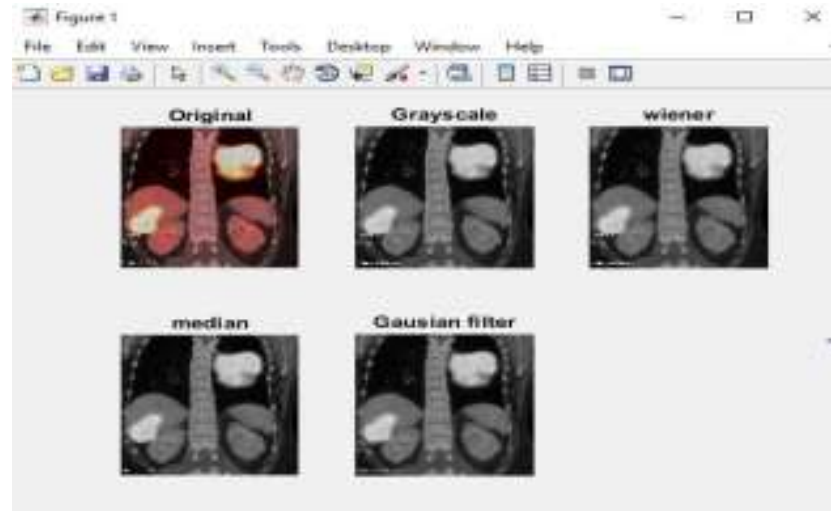

Figure 4.1 Filter

Figure 4.1, shows the output of the filtered image. After that the image is filtered to remove noise and other artifacts by using three filters such as median, wiener and gaussian filter. The performance evaluation for these filters using MSE and PSNR are tabulated below.
Table 4.1 Comparison of MSE

\begin{tabular}{|l|l|l|l|}
\hline Filterlimage & Image1 & Image2 & Image3 \\
\hline Median & 0.08 & 0.0081 & 0.0060 \\
\hline Wiener & 0.00046 & 0.00014 & 0.0015 \\
\hline Gaussian & 0.0059 & 0.0057 & 0.0043 \\
\hline
\end{tabular}

Table 4.1 shows the values of Mean square error and the variation is plotted using bar graph and it is shown below.

Table 4.2 Comparison of PSNR

\begin{tabular}{|l|c|c|c|}
\hline Filterlimage & Image1 & Image2 & Image3 \\
\hline Median & 68.7481 & 69.0720 & 70.3626 \\
\hline Wiener & 91.4645 & 90.4388 & 92.5220 \\
\hline Gaussian & 70.4465 & 70.5591 & 71.7665 \\
\hline
\end{tabular}

Table 4.2, shows the PSNR value and the variation is plotted in bar graph and it is shown below

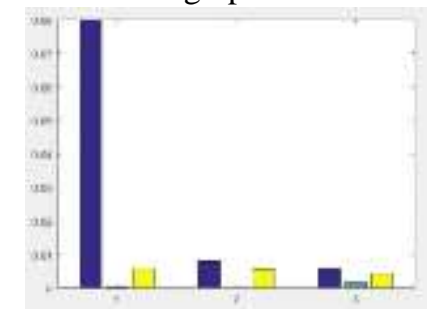

Figure 4.2 MSE

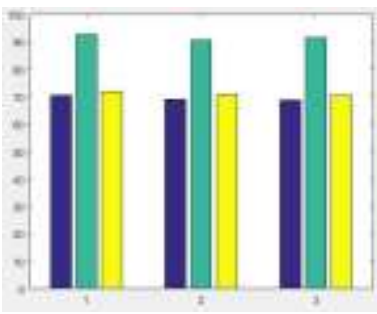

Figure 4.3 PSNR Figure
4.6 shows the plot for Mean square Error, it is clear that the values are very low for Wiener filter and Figure 4.7 shows the plot for PSNR, the values are very high for Wiener filter. From this it is clear that Wiener is Better than other two filters, which removes the additive noise.

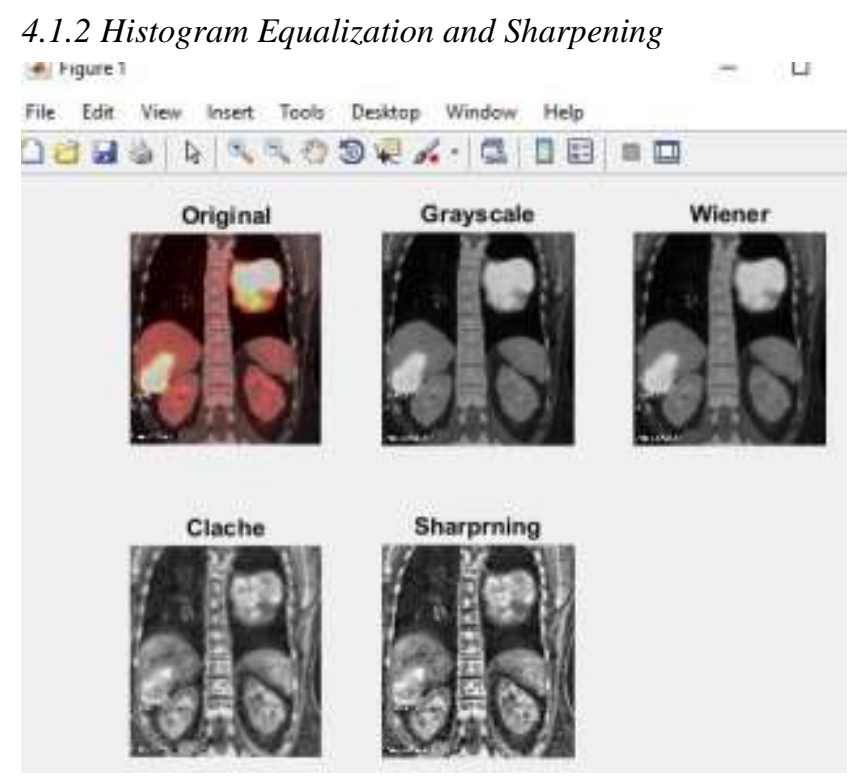

Figure 4.4 Histogram equalization and Sharpening

Figure 4.4 shows the histogram equalization by CLAHE and the unsharp masking of an image. The contrast of the image is enhanced, so that the intensity is distributed equally and the edges are enhanced by the high pass filter. 


\subsection{SEGMENTATION}

\subsubsection{Watershed Segmentation}

Figure 4.5, shows the output of watershed segmentation. The image is first converted in binary, then the binarized image is complemented finally the tumor is segmented using watershed transform. This watershed segmentation causes oversegmentation. It also segments the non tumor area. The boundary of the tumor is not segmented properly, the non tumor area is also segmented.

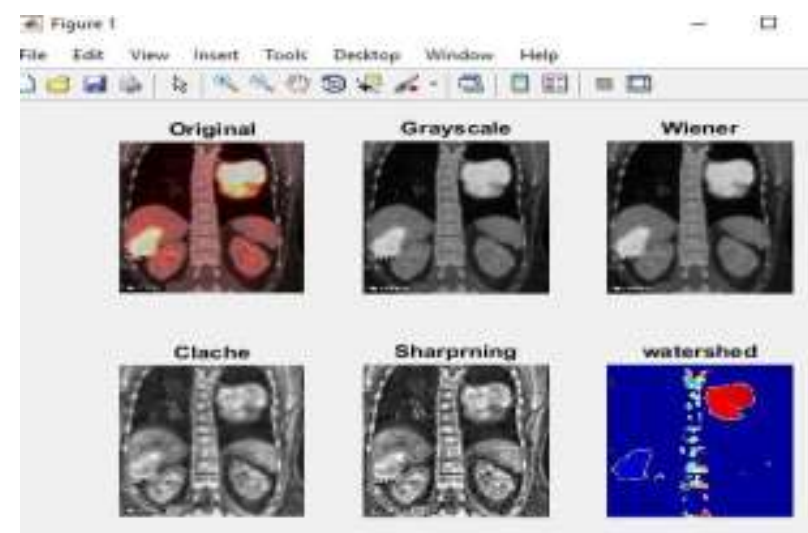

Figure 4.5 Watershed segmentation

\subsubsection{Thresholding Segmentation}

Figure 4.6 shows the output for Thresholding Segmentation. The pixel which is greater than the threshold is turned to white, and the pixels which is less than the threshold turned to black. This is an automatic thresholding segmentation. It is based on the Histogram of the Image. It will automatically calculates the histogram and perform segmentation.

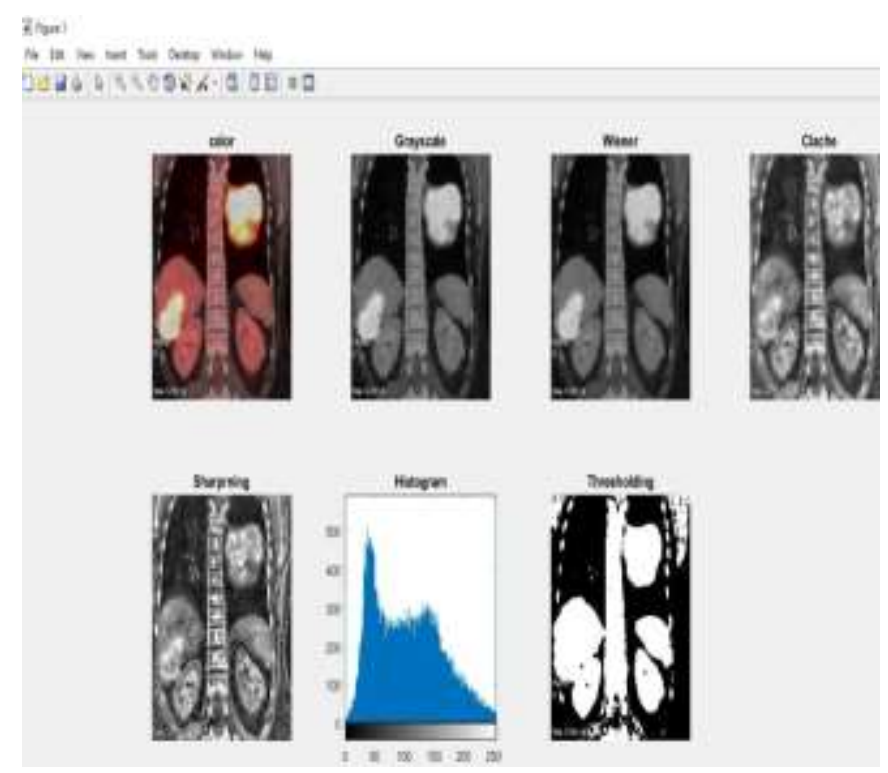

Figure 4.6 Thresholding Segmentation

\subsubsection{K-means Clustering Segmentation}

Figure 4.7, shows the output of k-means clustering segmentation. Every point of the data set is assigned to its nearest centroid and the centroid is moved toward the center of its assigned points. The $k$-means clustering algorithm works by finding like groups based on Euclidean distance, a measure of distance or similarity. The iteration continues untill centroid is shifted. Kmeans segmentation shows good result when compared to watershed and thresholding segmentation. $\mathrm{K}$ value is given as 2 .
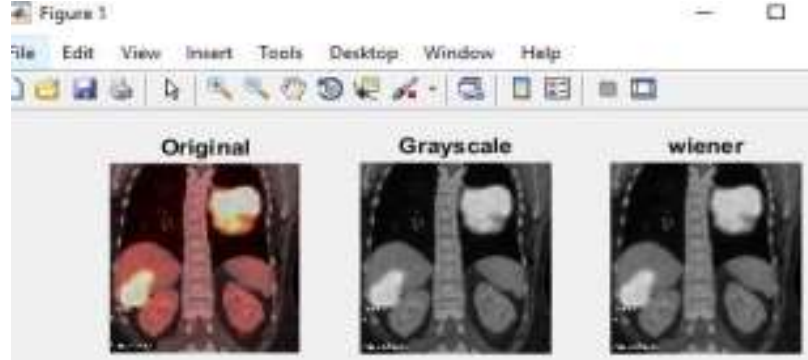

Clache
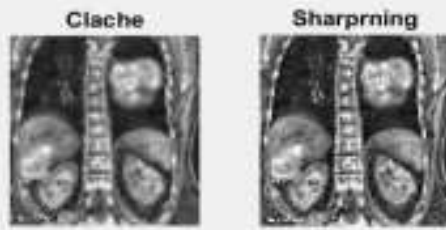

kmeans

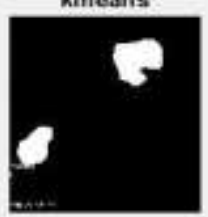

Figure 4.7 K-means Segmentation

\subsubsection{FCM clustering segmentation}

Figure 4.8 shows the output of FCM segmentation. The membership function maps each element of $X$ to a membership value between 0 and 1 . Triangular membership function is used here. In Fuzzy c means clustering the segmented part cannot be seen clearly. For that reasons, thresholding is applied to extract the segmented image portion. Like wise the output of FCM segmentation for other images is shown in figures.

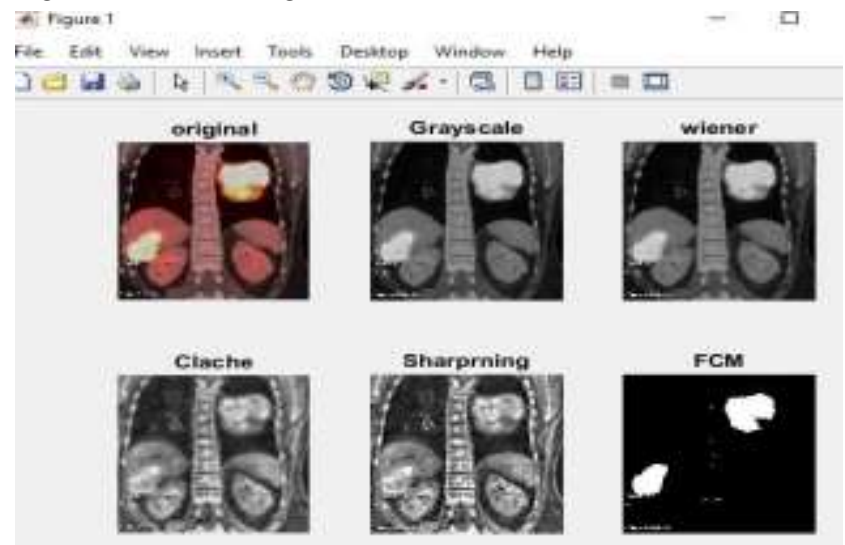

Figure 4.8 FCM clustering segmentation

\subsubsection{Mean shift Clustering Segmentation}

Figure 4.8 shows the output for Mean shift clustering segmentation. This is a Graph based segmentation. This is the unsupervised clustering segmentation. It finds the local Maxima of an image using probability distribution. Probability distribution is built using kernel estimator. The pixels which are under the local maxima are given to the kmeans to do segmentation. 
Ev rigure I

File Edit View Insert Tools Desktop Window Help

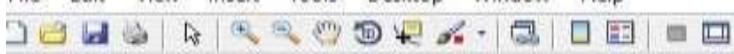
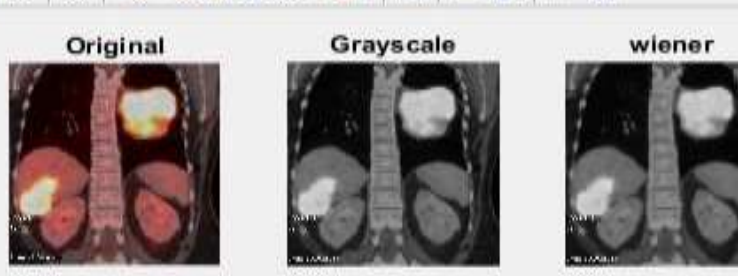

Clache
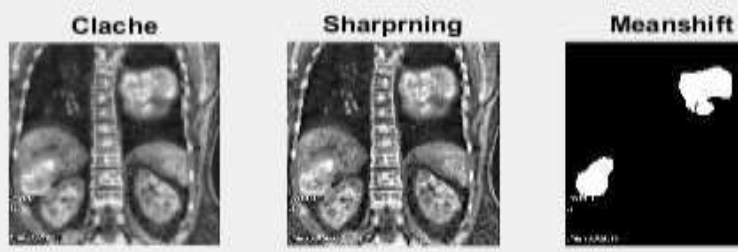

Figure 4.9 Mean shift segmentation

\subsubsection{Graph cut segmentation}

Figure 4.10 shows the output of Graph cut Segmentation. First the image is transformed into mapping function. All the pixels are considered as labels, this is to reduce the euclidean distance. After that it is given for graph cut segmentation. This gives the multiple partitionings on superpixels. All edges in the pairwise superpixel graph from the first layer are incorporated into the pairwise edge, other layer edges are incorporated as like first layer. The graph partition is shown in figure with the markings in an image.

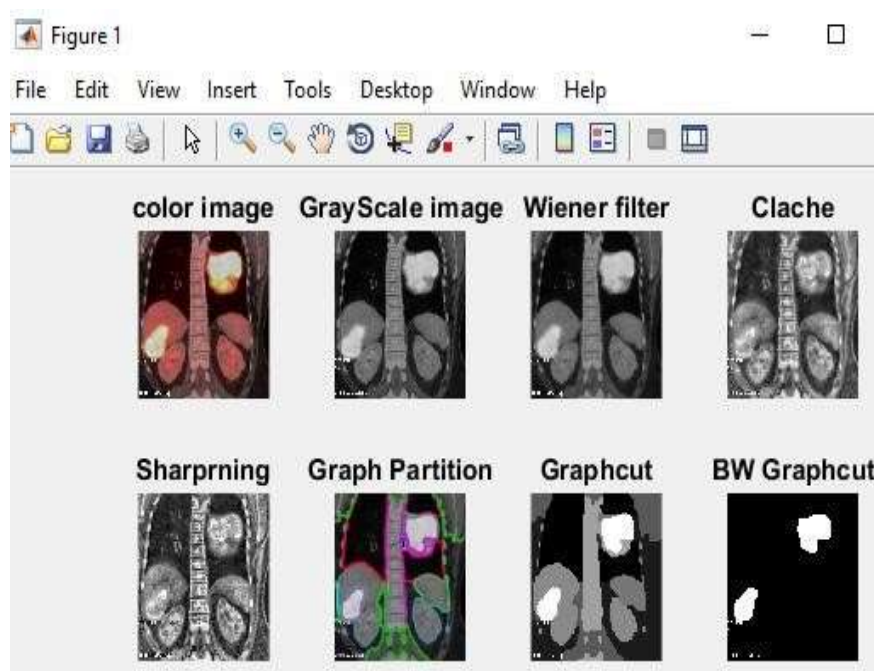

Figure 4.10 Graph cut segmentation

\subsubsection{Performance Evaluation of Segmentation}

Performance Evaluation for the segmentation was performed against the Ground Truth. The boundary of the ground truth and the segmented image should be the same for the perfect segmentation. Ground Truth for each image is shown in Figure and the evaluation parameters are tabulated below.
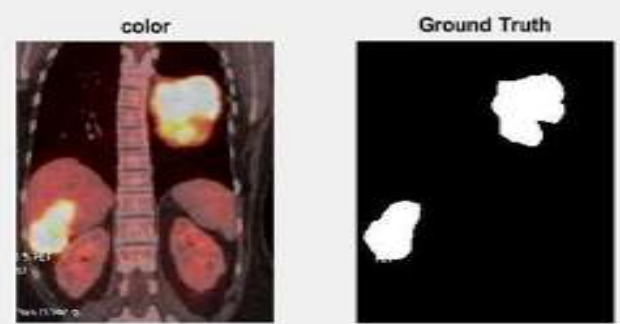

Figure 4.11 Ground Truth

Table 4.3 Jaccard Distance

\begin{tabular}{|l|l|l|l|l|l|l|}
\hline $\mathbf{I}$ & $\mathbf{W}$ & $\mathbf{T}$ & $\mathbf{K}$ & $\mathbf{F}$ & $\mathbf{M}$ & $\mathbf{G}$ \\
\hline $\mathbf{1}$ & 0.3142 & 0.2149 & 0.652 & 0.70 & 0.5557 & 0.715 \\
\hline $\mathbf{2}$ & 0.5142 & 0.7424 & 0.768 & 0.78 & 0.6860 & 0.825 \\
\hline $\mathbf{3}$ & 0.5177 & 0.2108 & 0.722 & 0.84 & 0.8503 & 0.931 \\
\hline $\mathbf{4}$ & 0.0501 & 0.8283 & 0.902 & 0.85 & 0.6293 & 0.783 \\
\hline $\mathbf{5}$ & 0.5028 & 0.2947 & 0.061 & 0.40 & 0.6935 & 0.875 \\
\hline $\mathbf{6}$ & 0.0122 & 0.0260 & 0.396 & 0.05 & 0.3968 & 0.377 \\
\hline $\mathbf{7}$ & 0.4193 & 0.0681 & 0.062 & 0.30 & 0.3424 & 0.602 \\
\hline
\end{tabular}

I-Image, W-Watershed, T-Threshold, K-Kmeans, F-FCM, M-Mean shift, G-Graph cut

Table 4.4 Shows the values of jaccard distance for all the images of all segmentation.

Table 4.4 Dice Similarity Coeficient

\begin{tabular}{|l|l|l|l|l|l|l|}
\hline $\mathbf{I}$ & $\mathbf{W}$ & $\mathbf{T}$ & $\mathbf{K}$ & $\mathbf{F}$ & $\mathbf{M}$ & $\mathbf{G}$ \\
\hline $\mathbf{1}$ & 0.3142 & 0.2149 & 0.6524 & 0.701 & 0.5557 & 0.7159 \\
\hline $\mathbf{2}$ & 0.6792 & 0.8572 & 0.8691 & 0.880 & 0.8137 & 0.9042 \\
\hline $\mathbf{3}$ & 0.6822 & 0.3482 & 0.8386 & 0.914 & 0.9191 & 0.9646 \\
\hline $\mathbf{4}$ & 0.0955 & 0.9061 & 0.9021 & 0.924 & 0.7725 & 0.7832 \\
\hline $\mathbf{5}$ & 0.6691 & 0.4553 & 0.1154 & 0.576 & 0.8190 & 0.9047 \\
\hline $\mathbf{6}$ & 0.0241 & 0.0506 & 0.0517 & 0.051 & 0.3968 & 0.3778 \\
\hline $\mathbf{7}$ & 0.5909 & 0.1274 & 0.1179 & 0.461 & 0.5102 & 0.7522 \\
\hline
\end{tabular}

I-Image, W-Watershed, T-Threshold, K-Kmeans, F-FCM, M-Mean shift, G-Graph cut

Table 4.4 Shows the values of dice similarity coefficient for all the images of all segmentation. Dice similarity coefficient value should high. If it is equal to 1 , then the Ground Truth Exactly matches the segmented image.

Table 4.5 Relative False Positive

\begin{tabular}{|l|l|l|l|l|l|l|}
\hline $\mathbf{I}$ & $\mathbf{W}$ & $\mathbf{T}$ & $\mathbf{K}$ & $\mathbf{F}$ & $\mathbf{M}$ & $\mathbf{G}$ \\
\hline $\mathbf{1}$ & 0.2924 & 0.0236 & 0.3271 & 0.24 & 0.7014 & 0.3108 \\
\hline $\mathbf{2}$ & 0.4132 & 0.0308 & 0.0261 & 0.07 & 0.3567 & 0.0905 \\
\hline $\mathbf{3}$ & 0.6616 & 0.0062 & 0.0148 & 0.03 & 0.0331 & 0.0307 \\
\hline $\mathbf{4}$ & 0.6109 & 0.0696 & 0.0770 & 0.07 & 0.4103 & 0.1185 \\
\hline $\mathbf{5}$ & 0.0128 & 0.0257 & 0.0021 & 0.00 & 0.0171 & 0.1576 \\
\hline $\mathbf{6}$ & 0.3876 & 0.0045 & 0.0012 & 0.00 & 0.0932 & 0.1513 \\
\hline $\mathbf{7}$ & 0.3144 & 0.0257 & 0.0311 & 0.31 & 0.2935 & 0.4102 \\
\hline
\end{tabular}

I-Image, W-Watershed, T-Threshold, K-Kmeans, F-FCM, M-Mean shift, G-Graph cut

Table 4.5 shows the value of Relative false positive. Relative false Positive gives the value of positive result, in the place of Negative result. The value of Relative false positive should be less. The table shows the value of RFP for all the segmentation. 
Table 4.6 Relative False Negative

\begin{tabular}{|l|l|l|l|l|l|l|}
\hline $\mathbf{I}$ & $\mathbf{W}$ & $\mathbf{T}$ & $\mathbf{K}$ & $\mathbf{F}$ & $\mathbf{M}$ & $\mathbf{G}$ \\
\hline $\mathbf{1}$ & 0.5939 & 0.780 & 0.1343 & 0.06 & 0.0546 & 0.0616 \\
\hline $\mathbf{2}$ & 0.2733 & 0.234 & 0.2115 & 0.15 & 0.0694 & 0.1001 \\
\hline $\mathbf{3}$ & 0.1398 & 0.787 & 0.2672 & 0.13 & 0.1216 & 0.0398 \\
\hline $\mathbf{4}$ & 0.9193 & 0.114 & 0.1152 & 0.07 & 0.1125 & 0.1240 \\
\hline $\mathbf{5}$ & 0.4908 & 0.705 & 0.9372 & 0.59 & 0.2946 & 0.2710 \\
\hline $\mathbf{6}$ & 0.9831 & 0.974 & 0.9735 & 0.94 & 0.5663 & 0.5651 \\
\hline $\mathbf{7}$ & 0.4489 & 0.930 & 0.9354 & 0.60 & 0.5570 & 0.1499 \\
\hline
\end{tabular}

I-Image, W-Watershed, T-Threshold, K-Kmeans, F-FCM, M-Mean shift , G-Graph cut

Table 4.6 shows the output of Relative False Negative. Relative false Negative give the values of Negative result, where we should get a Positive result. It should be less.

\subsection{FEATURE EXTRACTION}

The same image may contain malignant as well as benign. The variation for the features are obtained in mean and contrast. Contrast indicates the intensity of an image. Intensity of the benign is less when compared to malignant because of FDG uptake. Mean also depends on intensity. The image is converted into double before extracting features.

Table 4.7 Features

\begin{tabular}{|c|c|c|c|c|c|c|c|}
\hline \multicolumn{2}{|c|}{ mage } & C & Co & $\mathbf{E}$ & H & $\mathbf{M}$ & V \\
\hline \multirow[t]{2}{*}{1} & T1 & 0.0428 & 0.9842 & 0.9109 & 0.9957 & 0.0332 & 0.0013 \\
\hline & $\mathrm{T} 2$ & 0.0432 & 0.9728 & 0.9498 & 0.999 & 0.0188 & 0.144 \\
\hline \multirow{3}{*}{2} & $\mathrm{~T} 1$ & 0.0192 & 0.9931 & 0.9141 & 0.998 & 0.0336 & 0.0015 \\
\hline & $\mathrm{T} 2$ & 0.0338 & 0.9945 & 0.8103 & 0.9967 & 0.0795 & 0.0039 \\
\hline & T3 & 0.016 & 0.993 & 0.927 & 0.9983 & 0.0279 & 0.1783 \\
\hline 3 & TI & 0.03 & 0.9948 & 0.8115 & 0.9962 & 0.0757 & 0.0033 \\
\hline \multirow[t]{2}{*}{4} & $\mathrm{~T} 1$ & 0.033 & 0.995 & 0.8568 & 0.9976 & 0.0683 & 0.0054 \\
\hline & $\mathrm{T} 2$ & 0.0092 & 0.9863 & 0.9794 & 0.9995 & 0.0079 & 0.055 \\
\hline \multirow[b]{2}{*}{5} & $\mathrm{~T} 1$ & 0.0068 & 0.969 & 0.993 & 0.9995 & 0.0026 & 0.045 \\
\hline & $\mathrm{T} 2$ & 0.0322 & 0.9916 & 0.86 & 0.9971 & 0.0523 & 0.0021 \\
\hline 6 & T1 & 0.0095 & 0.9814 & 0.9841 & 0.9994 & 0.0059 & 0.032 \\
\hline \multirow[b]{2}{*}{7} & $\mathrm{~T} 1$ & 0.0310 & 0.9766 & 0.9542 & 0.9945 & 0.0164 & 0.0118 \\
\hline & $\mathrm{T} 2$ & 0.0679 & 0.7908 & 0.9864 & 0.9976 & 0.0042 & 0.030 \\
\hline
\end{tabular}

C-Contrast, Co-Correlation, E-Energy, H-Homogenity, M-Mean, V-Variance.

Table 4.7 shows the various feature values of the the tumors which vary with different intensities.

\subsection{CLASSIFICATION}

\subsubsection{KNN Classifier}

Features from the segmented image is trained using KNN Classifier. The testing is performed by comparing the values of test features with the trained features. Figure 4.11 shows the output of KNN Classifier.

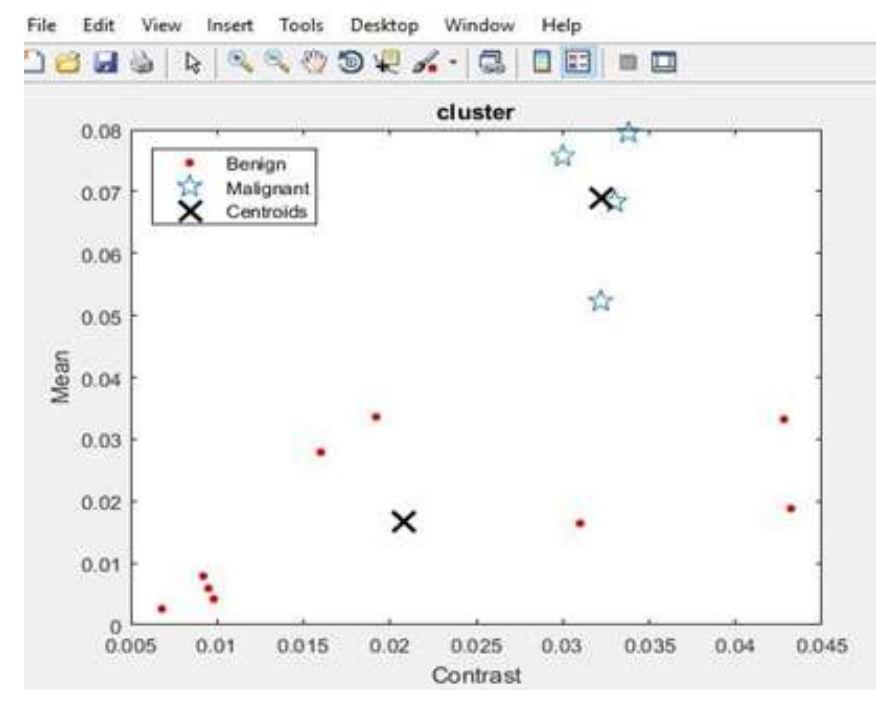

Figure 4.12 KNN Classifier

\subsubsection{SVM Classifier}

Features from the segmented image is trained using SVM Classifier. The testing is performed by comparing the values of test features with the trained features. Figure 4.12 shows the output of SVM classifier.

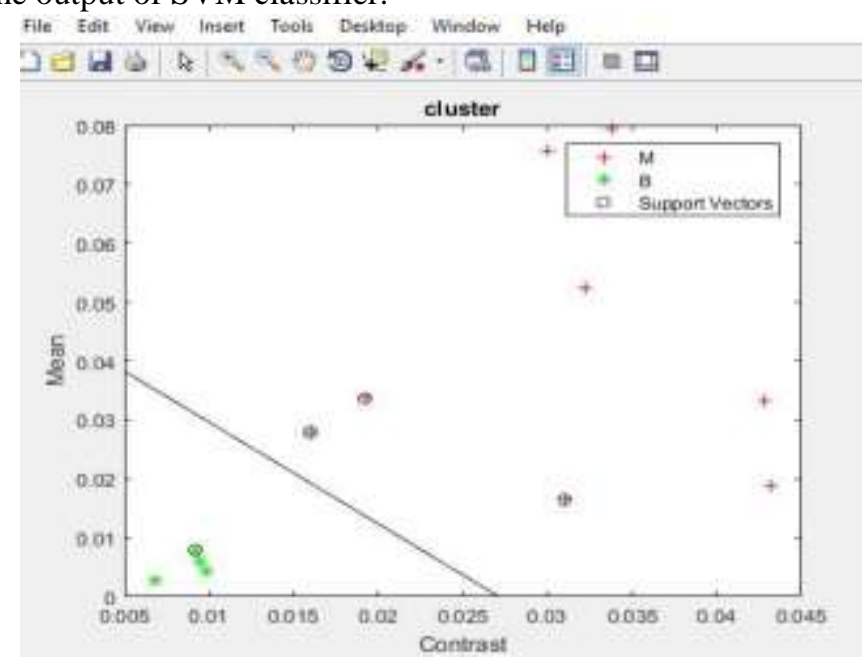

Figure 4.13 SVM classifier

when the test is performed against the trained values, if the tumor is benign the output will be displayed as in figure 4.13 , if the tumor is malignant the output will be displayed as in figure 4.14 .

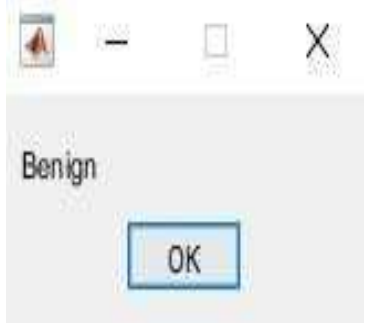

Figure 4.14 Benign

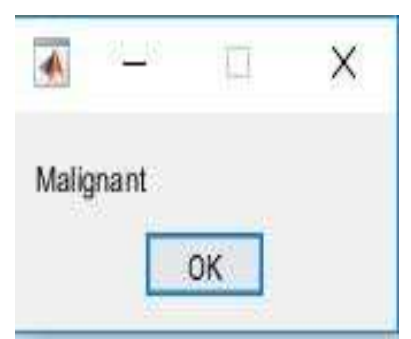

Figure 4.15 Malignant 
Table 4.8 Performance Measure of Classifier

\begin{tabular}{|l|l|l|}
\hline Classifier & KNN & SVM \\
\hline Mean & $89.5 \%$ & $96.5 \%$ \\
\hline Contrast & $87.5 \%$ & $95.5 \%$ \\
\hline
\end{tabular}

Table 4.8 shows the performance Measure of classifier. The accuracy value should be higher, from the table, the accuracy value of SVM is high when compared to KNN classifier. Thus the SVM classifier classifies the Malignant and Benign Tumor Reliably.

\section{CONCLUSION AND FUTURE WORK}

\subsection{CONCLUSION}

The overall objective of this project is to develop a algorithm to automatically detect the multiple tumors from lungs. The proposed algorithm preprocessed and segmented the tumor region from the PET/CT images. The features are extracted from the segmented image and the tumor is classified as the Normal FDG uptake and cancer tumors.

From the preprocessing it is concluded that wiener filter is best when compared to gaussian and median filter. The image is enhanced using CLAHE and unsharp masking to enchance the tumor part. Segmentation was performed using Watershed segmentation, Thresholding segmentation, Kmeans clustering segmentation, FCM clustering segmentation, Mean shift clustering segmentation and Graph cut segmentation. Evaluation metrics was calculated for the segmented image against the Ground Truth. Dice similarity coeffient and jaccard distance is high for the graph cut segmentation. Hence graph cut segmentation is the best segmentation. Features are extracted from the graph cut segmenation and then classified using SVM classifier and KNN classifier. Accuracy found in SVM classifier.

\subsection{Future Work}

The method of feature extraction from the image which contains multiple tumors can be selected automatically without any manual interpretation

\section{REFERENCES}

1. Appudurai Ramalingam, Somaskandan Suthakar. (2016) 'Estimating And Locating The Volumes Of Multiple Primary Non-small Cell Stage-i Bronchogenic Carcinomas From Medical Images Using Specially Devised Morphological Operations', IEEE, International Conference on Information Science (ICIS) .

2. Gawade Prathamesh, Chauhan R.P. (2016) 'Detection of Lung Cancer cells using Image processsing Techniques',IEEE, International Conference on Power Electronics. Intelligent Control and Energy Systems.

3. Neha Bangara and Akash Deep. (2014) 'Tumor Detection by Color Segmentation of PET/CT Liver Images', Journal Of Emerging Technologies In Web Intelligence, Vol. 6, No. 1.

4. Ning Guo, Ruoh-Fang Yen, Georges El Fakhri and Quanzheng Li. (2015) 'SVM Based Lung Cancer Diagnosis sing Multiple Image Features in PET/CT', IEEE.

5. Nivetha P. and Manikavasagam P. (2014) 'Lung cancer detection at early stage using PET/CT Imaging technique', International Journal of Innovative Research in Computer and Communication Engineering, Vol. 2, Issue 3.

6. Punithavathy K., Ramya M.M. and Sumathi Poobal (2015) 'Analysis of Statistical Texture Features for Automatic Lung Cancer Detection in PET/CT Images', international conference on Robotics, Automation, Control, embedded systems.

7. Srikanth B. and Sreenivasa Reddy E. (2016) 'Analysis and Detection of Multi Tumor from MRI of Brain using Advance Adaptive Feature Fuzzy C-means (AAFFCM) Algorithm' Indian Journal of Science and Technology, Vol 9(43)

8. Wei Ju, Deihui Xiang, Bin Zhang, Lirong Wang, Ivica Kopriva, Xinjian Chen. (2015) 'Random Walk and Graph Cut for Co-Segmentation of Lung Tumor on PETCT Image' IEEE Transactions on Image Processing.

9. Zexi Yu and Francis M. Bui, Paul Babyn. (2015) 'A 3D Semi-Automated Co-Segmentation Method for Improved Tumor Target Delineation in 3D PET/CT Imaging' IEEE.

10. Mohammed Ben Salah, Amar Mitiche and Ismail Ben Ayed (2011) 'Multiregion Image Segmentation by Parametric Kernel Graph Cuts' IEEE Trnasactions on Image Processing, Vol 20(2).

11. Sunil Kumar, Ahirwar R.R, Jain Y.K. (2014) 'Efficient 3-class Fuzzy C-Means Clustering algorithm with Thresholding for Effective Medical Image Segmentation' International Journal of Emerging Technology and Advanced Engineering Vol 4(10).

12. Yong-mei Zhou, Sheng-yi Jiang, Mei-lin Yin (2008) 'A Region-based Image Segmentation Method with MeanShift Clustering Algorithm' IEEE. 